\title{
$\underline{\text { A role for Interleukins in ochronosis in a chondrocyte in vitro model of Alkaptonuria }}$
}

Mistry $\mathrm{JB}^{1}$, Jackson $\mathrm{DJ}^{2}$, Bukhari $\mathrm{M}^{2}$, Taylor $\mathrm{AM}^{1}$.

${ }^{1}$ Lancaster Medical School, Faculty of Health \& Medicine, Lancaster University, LA1 4YB

${ }^{2}$ University Hospitals of Morecambe Bay NHS Foundation Trust; Royal Lancaster Infirmary;

Lancaster, UK

\section{Corresponding author}

Dr Adam Taylor

Lancaster Medical School

Faculty of Health and Medicine

Lancaster University

Bailrigg

LA1 4YB

E: a.m.taylor@lancs.ac.uk

Email addresses of authors:

a.m.taylor@lancs.ac.uk

j.mistry@doctors.org.uk

danieljackson03@gmail.com

Marwan.Bukhari@mbht.nhs.uk

\section{Funding}

The authors would like to thank the AKU Society, the Rosetrees Trust and University Hospitals Morecambe Bay NHS Foundation Trust for funding. 


\section{$\underline{\text { Abstract }}$}

\section{Objectives}

Alkaptonuria is a rare autosomal recessive condition resulting from inability to breakdown homogentisic acid (HGA); an intermediate in tyrosine degradation. The condition has a triad of clinical features, the most damaging of which is ochronotic osteoarthropathy. HGA is elevated from birth but pigmentation takes many years. We hypothesise that interleukins play a role in initiation and progression of ochronotic osteoarthropathy.

\section{Methods}

C20/A4 cells were cultured and maintained in $9 \mathrm{~cm}$ petri dishes containing either HGA at $0.33 \mathrm{mM}$, a single interleukin (IL-1 $\beta$, IL-6 or IL-10) at $1 \mathrm{ng} / \mathrm{ml}$ or a combination of HGA and a single interleukin. Statistical analysis of pigment deposits and cell viability was performed using analysis of variance with Newman-Keuls post-test.

\section{$\underline{\text { Results }}$}

All cultures containing HGA showed a significant increase in pigment deposition compared to control and IL cultures alone. The cultures containing HGA and IL-6 showed a significant increase in pigment deposits compared to HGA alone. The cell viability counts across all cultures on day 10 demonstrated a significant decrease in cultures containing HGA compared to those which did not. There was no significant difference between cultures containing just HGA or those combined with an interleukin.

\section{$\underline{\text { Conclusions }}$}

This work demonstrates a role for cytokines present in the joint(s) in the pigmentation process, particularly IL-6 and that the presence of HGA in joint tissues appears more detrimental to chondrocytes than the presence of any of the interleukins found in response to joint injury, trauma and OA. This further supports the evidence that the arthropathy in alkaptonuria is much more severe and rapidly progressing.

\section{$\underline{\text { Keywords }}$}

Alkaptonuria, Interleukins, Osteoarthritis, Ochronosis, IL-6, Nitisinone.

\section{$\underline{\text { Running title }}$}


A role for interleukins in alkaptonuria

\section{$\underline{\text { Introduction }}$}

Alkaptonuria (AKU) is a rare genetic disorder of autosomal recessive inheritance. It results from the absence of homogentisate 1, 2 dioxygenase (HGD), the enzyme responsible for the breakdown of homogentisic acid (HGA); an intermediate in the tyrosine degradation pathway which is produced primarily by hepatocytes in the liver [1-3].

Absence of this enzyme results in a triad of clinical features. Firstly elevated levels of HGA is excreted in the urine from birth. This has been observed as early as 57 hours after birth [4] and persists throughout life. Overtime elevated levels of HGA in plasma results in an increase in the levels of HGA in most tissues, particularly collagenous tissues [5-7]. This presence of HGA in collagenous tissues causes the second clinical feature which is ochronosis. Ochronosis is the darkening, by polymerisation of the HGA in collagenous tissues to give a blue/dark ochre colour to tissues [8]. This second feature is usually evidence in the $3^{\text {rd }}$ decade of life and beyond [4]. The final symptom is a result of ochronosis within articular cartilages of weight bearing joints giving ochronotic osteoarthropathy. The presentation of ochronotic osteoarthropathy is described as mimicking osteoarthritis, but being rapidly progressing and earlier in onset $[8,9]$. Individuals with AKU have no reported shortening of life, but they have a severely affected quality of life with multiple joint replacements, cardiac co-morbidities and calculi in numerous tissues [6, 10-14].

There has been advances in understanding the end stage manifestations of AKU and its use as a model for documenting new advances in understanding "common" OA and other arthropathies is shedding new light on disease processes $[8,9,15]$. There also appears to be a potential therapy for AKU. Many potential therapies have been suggested $[4,16]$ but most have side effects. There is growing interest in Nitisinone, a triketone herbicide used primarily to treat Hereditary Tyrosinaemia type I $[17,18]$, but also with a huge potential in AKU [19,20]. Early work has shown its effectiveness at reducing urinary HGA [20-22].

Whilst there is promise in the therapy to prevent ochronosis there is still a gap in the understanding of ochronosis. It is clear that $\mathrm{pH}$ has an effect on the conversion of HGA to polymer [23], as urine goes dark from birth [4]. However the ochronosis, following conversion of HGA to polymer that occurs in tissues, particularly joint cartilages takes a number of years to occur [24]. This delay in pigmentation from ochronosis within joints 
suggests that there is protection within the collagenous tissues or a factor that is intrinsic to the joint in later life plays a role in the pigmentation process.

One factor that has been shown to be increased as far as ochronosis is concerned is the oxidative stress encountered by cells in AKU patient tissues [25-28]. However, the presence of HGA alone cannot be the sole factor in causing the pigmentation given HGA is present systemically, if not from birth, hours or days shortly after for many years prior to joint manifestations caused by ochronosis. There must be some other factor that causes the onset of pigmentation in these tissues.

There is still much to discover about the pathogenesis of AKU, and similarly OA. The majority of research focuses on the role of cartilage and chondrocytes in the initiation and development of the conditions. In OA, research has highlighted that the loss of proteoglycans and cleavage of type II collagen occurs on the surface of cartilage [29]. This makes the cartilage permeable by increasing water content, which in turn decreases the tensile strength of cartilage making it susceptible to erosion and degradation. However, in early OA, the chondrocytes have been found to exhibit a transient proliferative response, and increase the synthesis of cartilage matrix, as an early repair mechanism [30, 31]. In AKU, an alteration in the cartilage matrix strength and composition is also seen at end stage $[8,9]$, but what drives this is unclear.

What is clear is that development of OA and potentially AKU stems from the chondrocytes and their failure to maintain a balance between synthesis and degradation of extracellular matrix [32-36]. On examination of ochronotic surgical waste tissues the pigmentation is not uniform [8]. If HGA was the sole factor for pigmentation, given its systemic presence all joint tissues should be equally pigmented. In light of the patchy, non-uniform pigmentation a potential focussed local factor within certain areas of tissues may be responsible for initiating the pigmentation process. Local trauma in joints, either due to over exertion or trauma/damage may sufficiently disturb the equilibrium that (just about) existed prior to injury.

Chondrocytes respond to biochemical and biomechanical stimuli and in doing so produce anabolic factors and catabolic factors which can promote extracellular matrix formation or degradation, respectively. The mechanical and biological stresses within the joint stimulate the production of inflammatory cytokines. There is evidence that the chondrocytes are a source of production of cytokines, in particular interleukin 1 (IL-1 $\beta$ ) which in turn induces 
the expression of MMPs, which contribute to ECM degradation [37]. It is IL-1 $\beta$ and tumour necrosis factor (TNF- $\alpha$ ) that are heavily implicated in the initiation and progression of OA. They have a synergistic relationship and increase the production of prostaglandin E2 (PGE2) stimulating the activities of other inflammatory pathways.

Within a normal joint, cytokines and growth factors are produced in small quantities and released into synovial fluid to act on resident cells. In OA, and potentially AKU, this fine homeostatic balance is disturbed. Cytokines are categorised into 4 groups based on function; catabolic, regulatory, inhibitory and anabolic. IL-1 $\beta$ and TNF- $\alpha$ are known to be potent proinflammatory cytokines. They are produced in response to cell stress. In OA it is TNF- $\alpha$ that drives inflammation, but IL-1 $\beta$ that sustains it and promotes cartilage erosion, whilst also stimulating the induction of PGE2 by other inflammatory pathways. The chondrocytes respond to these pro-inflammatory cytokines by increasing the production of proteinases, prostaglandins and nitric oxide $[36,38,39]$.

IL-6 is classed as a pro-inflammatory cytokine. It predominantly modulates the effects of catabolic cytokines involved in cartilage destruction, and is often found in the synovial fluid of OA joints. It is produced by osteoblast cells in response to stimulation by IL-1 $\beta[40,41]$.

IL-10 is classed as an anti-inflammatory cytokine due to its ability in vitro to decrease the production and activity of catabolic cytokines [42]. This cytokine, is also found within the synovial fluid of OA joints. Interestingly IL-10 along with IL-4, in vivo, has been found to be chondroprotective. Testing in murine models of streptococcal cell wall arthritis, a combination of IL-10 and IL-4 was shown to be a potent down-regulator of the natural inflammatory response in OA [43]. In this paper we utilise one cytokine from 3 of the categories; IL-1 $\beta$, IL-6 and IL-10, either alone or in combination with HGA to examine the effects on cell viability and the pigmentation seen in ochronosis in AKU to highlight potential other contributory factors to the pigmentation process.

\section{Materials and Methods}

All reagents including HGA and Interleukins (IL-1 $\beta$, IL-6 and IL-10) were obtained from Sigma-Aldrich (Dorset, UK) unless otherwise stated. Plasticware was purchased from Appleton Woods (UK).

\section{Cell culture}


C20/A4 immortalized chondrocytes were a kind gift from Dr Mary B Goldring (Hospital for Special Surgery, NY, USA) [44]. C20/A4 cells were cultured and maintained in $9 \mathrm{~cm}$ petri dishes containing DMEM/Ham's F12 (1:1). The cells were maintained at $37^{\circ} \mathrm{C}$ in a humidified atmosphere of $5 \% \mathrm{CO}_{2}$. Medium was changed every 3 days. Medium was supplemented with HGA at concentrations of $0.33 \mathrm{mM}$, as previously described [24]. Interleukins were added to relevant wells at $1 \mathrm{ng} / \mathrm{ml}$.

\section{Identification and quantitation of ochronotic pigment deposition in vitro in the presence and absence of Interleukin's}

Cells were cultured and seeded as previously described with minor modifications for cell type (24). Specifically, C20/A4 cells were cultured in $9 \mathrm{~cm}$ petri dishes to confluence and then trypsinised and counted using a disposable C-Chip hemocytometer (Labtech International, UK), and light microscope. They were then seeded at a density of $4 \times 10^{4}$ cells/well into 24 well plates containing 13-mm diameter sterile glass coverslips. Medium was changed every $3^{\text {rd }}$ day and the supernatant spun down and stored at $-80^{\circ} \mathrm{C}$. Cells were allowed to grow for either 10 or 21-23 days. At these time points coverslips were washed 3 times with PBS solution in order to remove residual media and then were fixed using a 10\% PBFS solution. Coverslips were then stained with Schmorls reagent, rinsed in distilled water, counter stained with nuclear fast red, dehydrated in alcohols and mounted on a glass slide using DPX microscopy mountant [24].

Pigmentation, each deposit counted as a blue microscopic deposit within the field of view, was quantified by photographing 6 random areas on each slide and overlaying a grid on the image and then counting the number of pigment deposits/area and then calculating the average [24].

\section{Influence of HGA on cell growth and viability}

C20/A4 cells were seeded into $24-$ well plates at a concentration of $4 \times 10^{4}$ cells per well and grown for up to 10 days in $1 \mathrm{ml}$ of medium supplemented with $0.33 \mathrm{mM}$ HGA and $1 \mathrm{ng} / \mathrm{ml}$ of interleukin (either IL-1 $\beta$, IL-6 or IL-10).

Cell layers were washed, trypsinised and counted in disposable haemocytometers (Labtech International, UK). Trypan blue exclusion assay was used to determine viability.

\section{Statistical analysis}


Statistical analysis of pigment deposits (days 10 and 21-23) and cell viability (day 10) was performed using analysis of variance on Graphpad Prism 5 software (La Jolla, CA, USA). Differences between groups was determined by Newman-Keuls post-test.

\section{Ethical Approval}

This study was approved by Lancaster University Research Ethics Committee.

\section{$\underline{\text { Results }}$}

Cultures of C20/A4 cells with HGA added to them showed the characteristic darkening (data not shown), indicative of ochronosis as the result of polymerisation of HGA in both cultures with HGA and also those with the combination of HGA \& IL's, there was no obvious macroscopic difference in darkening between the two groups. There was also no obvious macroscopic difference in the darkened appearance of the media between the interleukin's (IL-1 $\beta$ vs IL-6 vs IL-10) on any given day, although those from day 21-23 were darker than those on day 10 (data not shown).

\section{(Figure 1 here)}

Both control and IL-1 $\beta$ supplemented cultures shown in (Figure 1) appear healthy and viable at both time points, as demonstrated by a continuous presence of C20/A4 cells when viewed during culture and histological examination. The cultures containing HGA, either alone or in combination with IL-1 $\beta$, contrasted to the controls, showed a decrease in the number of cells - but as expected an increased number of blue staining area indicative of pigment deposits. The staining indicating pigmentation was visible both intra- and extracellularly in the cultures containing HGA.

No pigment deposition was seen in the control and IL-1 $\beta$ cultures. There were statistically significant increases in the amount of pigment deposition in both cultures containing HGA, when compared to both control \& IL-1 $\beta$ alone. After 10 days in culture there was slightly more pigment deposits/unit area than HGA+IL-1 $\beta$, but this was not a significant increase. After 21 days there was more pigment deposits in the cultures containing HGA, but these were not significantly different from each other.

\section{(Figure 2 here)}

The viability of cells cultured with HGA, IL-1 $\beta$ or in combination demonstrated a significant decrease when compared to the control group. There was no significant difference between 
those containing HGA, both were significantly reduced compared to the control but not different to each other. Interestingly, the cultures containing HGA, either alone or in combination with IL-1 $\beta$ were significantly reduced compared to the culture containing only IL-1 $\beta$ (Figure 2). This is indicative of the effect of HGA being more detrimental to the viability of cells than the presence of IL-1 $\beta$.

(Figure 3 here)

The results from cultures containing HGA and IL-6 show that after 10 days the number of pigment deposits seen is significantly increased in those containing HGA+IL-6 compared to all other. On day 23 the number of pigment deposits per unit area increased compared to day 10 in both cultures containing HGA. On day 23 there was a significant increase in the number of pigment deposits in the HGA culture compared to the control. As with day 10, there was a significant increase in the number of pigment deposits per unit area in the culture containing HGA+IL-6 compared to HGA alone (Figure 3).

\section{(Figure 4 here)}

The viability of C20/A4 cells cultured in HGA and or IL-6 showed no significant difference between the control cells and those cultured in IL-6. The cultures containing HGA, both alone and with IL-6 were significant reduced compared to the control and IL-6 alone. There was no significant difference in the number of cells when comparing the cultures containing HGA; alone and with IL-6. The presence of HGA either alone, or in combination with IL-6 appears more detrimental to the cells than IL-6 alone (Figure 4).

(Figure 5 here)

The C20/A4 cultures with IL-10 for 10 days showed no significant differences across any of the cultures. By day 21 the number of pigment deposits in HGA only culture had increased, but those containing HGA+IL-10 had increased only slightly. By day 21 there was a highly significant increase in the number of pigment deposits in the HGA culture compared to the control and a significant increase in pigment deposits in the HGA+IL-10 compared to the control. The comparison of number of pigment deposits between the culture containing HGA and HGA+IL-10 showed no significant differences (Figure 5).

\section{(Figure 6 here)}

The effect of HGA and IL-10 on cell numbers showed that compared to the control IL-10 showed a small decrease in cell number, but this was not significant. Both cultures which 
contained HGA were significantly decreased compared to the control culture and that containing IL-10 alone. There was no significant difference between the cultures containing HGA and those containing HGA+IL-10.

\section{$\underline{\text { Discussion }}$}

This manuscript is the first to propose a possible role for another factor; IL-6, in the pigmentation process in AKU. HGA in joint tissues of patients with $\mathrm{AKU}$ is key, for the initiation and progression of the polymerisation process leading to arthritis and joint destruction in this condition. It has been demonstrated that HGA is present in AKU patients urine from birth [4], but the polymerisation seen in joint tissues does not seem to be immediate, as detailed by the years it takes for polymerisation to occur in vivo [24]. It may be that other factors, such as IL-6 may have a possible role in the pigmentation process. There is limited data or hypotheses in the published literature that elucidates any information on why the pigmentation process is not as quick as the dark colouration of urine. Given that individuals with AKU are as likely as other individuals without the condition to suffer from other age related joint conditions such as OA, the pigmentation process occurring as part of ochronotic osteoarthropathy may be marking cells which are damaged or undergoing age related changes.

It is known that IL-1 $\beta$ inhibits chondrocyte proliferation [45] and influences apoptosis in chondrocytes [46]. Our results show that the effect of IL-1 $\beta$ on chondrocytes is consistent with the published literature, a significant decrease in cell number compared to the control cultures. The effect of HGA on cell number is far more detrimental than that observed with IL-1 $\beta$ alone. This suggests that the effect HGA has is far more destructive to chondrocytes within the cartilage matrix. Interestingly the combination of HGA+IL-1 $\beta$ does not show a significant difference when compared to the HGA alone, this combination does show a significant decrease in the number of cells when compared to IL-1 $\beta$ alone. Initially, at day 10 the culture containing HGA alone shows more, although not significant, pigmentation compared to HGA+IL-1 $\beta$. After 21 days the combination culture shows more pigmentation than HGA alone. This may mean that longer exposure to IL-1 $\beta$ is needed to exert an effect on the pigmentation process. It has been shown that IL-1 $\beta$ is highest initially 24 hours after trauma, but can be elevated for weeks or months following the incident [47-52]. When considered in combination the results of the cell viability assay and pigmentation suggest that 
over time potentially fewer cells are responsible for the pigmentation process that is occurring, or the pigmentation that is occurring is not solely cellular in origin. However, the evidence suggests cells are key to driving the pigmentation process [24].

The pigmentation seen in cultures with HGA and IL-6 show that a combination of both shows a significant increase in pigmentation above that seen in HGA alone at both day 10 and day 23 ( $\mathrm{P}<0.01$ on both time points). Similarly the effect of HGA and IL-6 shows a significant decrease in cell viability when compared to both the control cultures and IL-6 alone. Interestingly there was no significant difference seen between the control and the IL-6 cultures. IL-6 has a role in OA, with a strong correlation between elevated IL-6 and OA [53, 54]. The effect of HGA on the cultures appears to be greater than that of IL-6 alone, with a combination of IL-6 and HGA showing similar viability, not significantly different, to that of HGA alone.

Interestingly, IL-10 with its chondroprotective properties $[55,56]$, did not seem to reduce the effects of HGA. The early (day 10) cultures of HGA+IL-10 show no significant difference compared to HGA alone. By day 21, the amount of pigmentation had increased in both cultures containing HGA, with the HGA showing a much larger increase compared to HGA+IL-10, which increased slightly, the difference between the two was not significant. This suggests that any potential protective effect from IL-10 is not seen immediately or in the short term (10 days). IL-10 may take longer to exert any chondroprotective effect in the presence of HGA and this may be why the 21 day culture of HGA+IL-10 had not increased at the same rate as HGA alone, any effect it may have is not significant compared to HGA. The presence of IL-10 has no significant effect on the pigmentation rate, mirroring the absence of significance between number of viable cells compared between HGA and HGA+IL-10.

IL-1 $\beta$ appears to have the most significant reduction/effect on cell number compared to control. This greater reduction in viable cells compared to IL-6 \& IL-10 vs their control, is potentially part of the reason why the arthropathy in AKU is faster and of a more rapid onset than that seen in the more common OA. The condition is often mis-diagnosed as OA $[4,57$, 58], but little is known about the potential overlap of physiological disease processes. Evidence exists in the literature characterising the more severe and rapidly progressing anatomicopathological changes in joint tissues of AKU sufferers compared to OA [8].

The presence of HGA in AKU tissues has been shown to increase oxidative stress on cells [25-27,59], this involvement of changes in the antioxidant defences is also seen in OA with 
both IL-1 $\beta$ and IL-6 shown to dysregulate antioxidant defences in chondrocytes [60]. The significance of the IL-6 results presented in this study, showing a significant increase in pigmentation compared to HGA and IL-6 alone presented in this study are supported by clinical evidence that there is a significant increase in serum IL-6 in AKU patients compared to control [61]. Previous in vitro work has shown that chondrocytes challenged with HGA show a significant increase in pro-inflammatory cytokines, with IL-6 being the most significantly increased [62]. This study also demonstrated that the AKU patients had higher serum levels of C-reactive protein and IL-1 $\beta$ although this was not significant [61].

Our results further support the theory that regardless of the presence of any of the interleukins, their effect seems less detrimental to cultures of chondrocytes compared to that of HGA. In the clinical setting this suggests that the most beneficial strategy for patients would be to target the reduction or eradication of HGA from tissues and plasma to minimise the chance of pigmentation and the effect the HGA has on cells.

There is currently new hope for sufferers of AKU, with the trial of Nitisinone in patients with the condition [19], the elimination of HGA from the body will prevent the pigmentation process in individuals. However, there are many sufferers, particularly in the developing world who will not have access to this treatment and need to reduce their risk of pigmentation at an early age. These patients could benefit from avoiding over exertion and traumatic insult on joint tissues and the production of cytokines that have the potential to reduce cell number in combination with HGA and increase the rate of pigmentation, leading to joint disease, failure and ultimate need for replacement. In individuals that are taking Nitisinone, there is the possibility of complications and the therapy may need to be halted or removed completely. Some patients report elevated plasma tyrosine levels and corneal opacities [21], but on the whole Nitisinone appears relatively well tolerated.

Our cultures utilised a concentration of HGA that has been tested previously in in vitro models of $\mathrm{AKU}$, but more importantly is physiologically within the range seen in plasma of patients with AKU [24]. This raises the question of what other potential factors may be acting within cartilaginous tissues that are affected by pigmentation in alkaptonuric patients and how these may be understood to prevent pigmentation in individuals who do not have access to the promising and effective therapeutic agent Nitisinone[19, 63]. Results presented in this study show that IL-6 may be partly responsible for an increase in pigmentation compared to the HGA alone, but what promotes the presence of IL-6 at an increased level in AKU patients 
still needs further investigation [61]. Is it the presence of HGA or its polymeric derivatives that increases the oxidative stresses and drives inflammation, or are inflammatory cytokines released in response to trauma and this initiates and drives the pigmentation process?

\section{$\underline{\text { Key Messages }}$}

HGA is more detrimental to chondrocyte viability than interleukins hence alkaptonuria arthropathy is more severe.

IL-6 appears to be involved in increasing ochronotic pigmentation in alkaptonuria

IL-1 $\beta$ and IL-10 do not appear to affect ochronotic pigmentation in alkaptonuria.

\section{Conflict of interest}

The authors declare no conflict of interest.

\section{$\underline{\text { References }}$}

1. Fisher AA, Davis MW. Alkaptonuric ochronosis with aortic valve and joint replacements and femoral fracture. Clin Med Res. 2004;2:209-15.

2. Fernandez-Canon JM, Granadino B, de Bernabe DBV, Renedo M, Fernandez-Ruiz E, Penalva MA, et al. The molecular basis of alkaptonuria. Nat Genet. 1996;14:19.

3. La Du BN, Zannoni VG, Laster L, Seegmiller JE. The nature of the defect in tyrosine metabolism in alcaptonuria. J Biol Chem. 1958;230:251.

4. Mistry JB, Bukhari M, Taylor AM. Alkaptonuria. Rare Dis. 2013 Dec 18;1:e27475. doi: 10.4161/rdis.27475.

5. Taylor AM, Wlodarski B, Prior IA, Wilson PJ, Jarvis JC, Ranganath LR, et al. Ultrastructural examination of tissue in a patient with alkaptonuric arthropathy reveals a distinct pattern of binding of ochronotic pigment. Rheumatology (Oxford). 2010 Jul;49(7):1412-4.

6. Taylor AM, Wilson PJ, Ingrams DR, Helliwell TR, Gallagher JA, Ranganath LR. Calculi and intracellular ochronosis in the submandibular tissues from a patient with alkaptonuria. $\mathbf{J}$ Clin Pathol. 2010 Feb;63(2):186-8.

7. Helliwell TR, Gallagher JA, Ranganath L. Alkaptonuria--a review of surgical and autopsy pathology. Histopathology. 2008 Nov;53(5):503-12. 
8. Taylor AM, Boyde A, Wilson PJ, Jarvis JC, Davidson JS, Hunt JA, et al. The role of calcified cartilage and subchondral bone in the initiation and progression of ochronotic arthropathy in alkaptonuria. Arthritis Rheum. 2011 Dec;63(12):3887-96.

9. Taylor AM, Boyde A, Davidson JS, Jarvis JC, Ranganath LR, Gallagher JA. Identification of trabecular excrescences, novel microanatomical structures, present in bone in osteoarthropathies. Eur Cell Mater. 2012 Apr 21;23:300-9.

10. Araki K, Sudo A, Hasegawa M, Uchida A. Devastating ochronotic arthropathy with successful bilateral hip and knee arthroplasties. J Clin Rheumatol. 2009 Apr;15(3):138-40.

11. Pettit SJ, Fisher M, Gallagher JA, Ranganath LR. Cardiovascular manifestations of Alkaptonuria. J Inherit Metab Dis. 2011 Dec;34(6):1177-81.

12. Phornphutkul C, Introne WJ, Perry MB, Bernardini I, Murphey MD, Fitzpatrick DL, et al. Natural history of alkaptonuria. N Engl J Med. 2002 Dec 26;347(26):2111-21.

13. Sridhar FK, Mukha RP, Kumar S, Kekre NS. Lower urinary tract symptoms and prostatic calculi: A rare presentation of alkaptonuria. Indian J Urol. 2012 Apr;28(2):219-21.

14. Krízek V. Urolithiasis and prostatolithiasis in alcaptonuria with ochronosis. Int Urol Nephrol. 1971;3(3):245-50

15. Boyde A, Davis GR, Mills D, Zikmund T, Cox TM, Adams VL, et al. On fragmenting, densely mineralised acellular protrusions into articular cartilage and their possible role in osteoarthritis. J Anat. 2014 Oct;225(4):436-46.

16. Ranganath LR, Jarvis JC, Gallagher JA. Recent advances in management of alkaptonuria. J Clin Pathol. 2013 May;66(5):367-73.

17. Lindstedt S, Holme E, Lock EA, Hjalmarson O, Strandvik B. Treatment of hereditary tyrosinaemia type I by inhibition of 4-hydroxyphenylpyruvate dioxygenase. Lancet. 1992 Oct 3;340(8823):813-7.

18. Lock EA, Ellis MK, Gaskin P, Robinson M, Auton TR, Provan WM, et al. From toxicological problem to therapeutic use: the discovery of the mode of action of 2-(2-nitro-4trifluoromethylbenzoyl)-1,3-cyclohexanedione (NTBC), its toxicology and development as a drug. J Inherit Metab Dis. 1998 Aug;21(5):498-506.

19. Ranganath LR, Milan AM, Hughes AT, Dutton JJ, Fitzgerald R, Briggs MC, et al. Suitability Of Nitisinone In Alkaptonuria 1 (SONIA 1): an international, multicentre, 
randomised, open-label, no-treatment controlled, parallel-group, dose-response study to investigate the effect of once daily nitisinone on 24-h urinary homogentisic acid excretion in patients with alkaptonuria after 4 weeks of treatment. Ann Rheum Dis. 2014 Dec 4. pii: annrheumdis-2014-206033. doi: 10.1136/annrheumdis-2014-206033.

20. Arnoux JB, Le Quan Sang KH, Brassier A, Grisel C, Servais A, Wippf J, et al. Old treatments for new insights and strategies: proposed management in adults and children with alkaptonuria. J Inherit Metab Dis. 2015 Apr 10.

21. Introne WJ, Perry MB, Troendle J, Tsilou E, Kayser MA, Suwannarat P, et al. A 3-year randomized therapeutic trial of nitisinone in alkaptonuria. Mol Genet Metab. 2011 Aug;103(4):307-14.

22. Gertsman I, Barshop BA, Panyard-Davis J, Gangoiti JA, Nyhan WL. Metabolic Effects of Increasing Doses of Nitisinone in the Treatment of Alkaptonuria. JIMD Rep. 2015 Feb 10. [Epub ahead of print]

23. Lodh M, Kerketta JA. Early diagnosis of co-existent $ß$-thalassemia and alkaptonuria. Indian J Hum Genet. 2013 Apr;19(2):259-61.

24. Tinti L, Taylor AM, Santucci A, Wlodarski B, Wilson PJ, Jarvis JC, et al. Development of an in vitro model to investigate joint ochronosis in alkaptonuria. Rheumatology (Oxford). $2011 \mathrm{Feb} ; 50(2): 271-7$.

25. Braconi D, Millucci L, Bernardini G, Santucci A. Oxidative stress and mechanisms of ochronosis in alkaptonuria. Free Radic Biol Med. 2015 Feb 27. pii: S0891-5849(15)00087-8. doi: 10.1016/j.freeradbiomed.2015.02.021.

26. Millucci L, Ghezzi L, Paccagnini E, Giorgetti G, Viti C, Braconi D, et al. Amyloidosis, inflammation, and oxidative stress in the heart of an alkaptonuric patient. Mediators Inflamm. 2014;2014:258471. doi: 10.1155/2014/258471.

27. Braconi D, Millucci L, Ghezzi L, Santucci A. Redox proteomics gives insights into the role of oxidative stress in alkaptonuria. Expert Rev Proteomics. 2013 Dec;10(6):521-35

28. Spreafico A, Millucci L, Ghezzi L, Geminiani M, Braconi D, Amato L, et al. Antioxidants inhibit SAA formation and pro-inflammatory cytokine release in a human cell model of alkaptonuria. Rheumatology (Oxford). 2013 Sep;52(9):1667-73. 
29. Poole AR, Kobayashi M, Yasuda T, Laverty S, Mwale F, Kojima T, et al. Type II collagen degradation and its regulation in articular cartilage in osteoarthritis. Ann Rheum Dis. 2002 Nov;61 Suppl 2:ii78-81.

30. Pearle AD, Warren RF, Rodeo SA. Basic science of articular cartilage and osteoarthritis. Clin Sports Med. 2005;24:1-12.

31. Goldring MB. Osteoarthritis and cartilage: The role of cytokines. Curr Rheumatol Rep. 2000;2:459-65.

32. Goldring MB. Update on the biology of the chondrocyte and new approaches to treating cartilage diseases. Best Pract Res Clin Rheumatol. 2006;20:1003-25.

33. Sovani S, Grogan SP. Osteoarthritis: Detection, pathophysiology, and current/future treatment strategies. Orthop Nurs. 2013;32:25-36.

34. Goldring MB, Berenbaum F. The regulation of chondrocyte function by proinflammatory mediators: prostaglandins and nitric oxide. Clin Orthop Relat Res. 2004;427:S37-46.

35. Goldring SR, Goldring MB. The role of cytokines in cartilage matrix degeneration in osteoarthritis. Clin Orthop Relat Res. 2004;427:S27 - 36.

36. Goldring MB, Marcu KB. Cartilage homeostasis in health and rheumatic diseases. Arthritis Res Ther. 2009;11:224.

37. Goldring MB, Otero M, Plumb DA, Dragomir C, Favero M, El Hachem K, et al. Roles of inflammatory and anabolic cytokines in cartilage metabolism: signals and multiple effectors converge upon MMP-13 regulation in osteoarthritis. "Eur Cell Mater. 2011 Feb 24;21:20220.

38. Dayer JM. The process of identifying and understanding cytokines: from basic studies to treating rheumatic diseases. Best Pract Res Clin Rheumatol. 2004;18:31-45.

39. Loeser RF. Molecular mechanisms of cartilage destruction in osteoarthritis. J Musculoskelet Neuronal Interact. 2008;8:303-6.

40. Westacott CI, Sharif M. Cytokines in osteoarthritis: Mediators or markers of joint destruction? Semin Arthritis Rheum. 1996;25:254-72. 
41. Takaoka Y, Niwa S, Nagai H. Interleukin-1B induces interleukin-6 production through the production of prostaglandin E2 in human osteoblasts, MG-63 cells. J Biochem. 1999;126:553-8.

42. Alaaeddine N, Di Battista JA, Pelletier J, Kiansa K, Cloutier J, Martel-Pelletier J. Inhibition of tumor necrosis factor \&alpha;-induced prostaglandin E2 production by the antiinflammatory cytokines interleukin-4, interleukin-10, and interleukin-13 in osteoarthritic synovial fibroblasts: Distinct targeting in the signaling pathways. Arthritis Rheum. $1999 ; 42: 710-8$.

43. Lubberts E, Joosten LAB, Helsen MMA, Van Den Berg WB. Regulatory role of interleukin 10 in joint inflammation and cartilage destruction in murine streptococcal cell wall (SCW) arthritis. More therapeutic benefit with IL-4/IL-10 combination therapy than with IL-10 treatment alone. Cytokine. 1998;10:361-9.

44. Goldring MB, Birkhead JR, Suen LF et al. Interleukin-1 beta-modulated gene expression in immortalized human chondrocytes. J Clin Invest 1994;94:2307-2316.

45. Blanco FJ, Lotz M. IL-1-induced nitric oxide inhibits chondrocyte proliferation via PGE2. Exp Cell Res. 1995 May;218(1):319-25.

46. López-Armada MJ, Caramés B, Lires-Deán M, Cillero-Pastor B, Ruiz-Romero C, Galdo $\mathrm{F}$, et al. Cytokines, tumor necrosis factor- $\alpha$ and interleukin- $1 \beta$, differentially regulate apoptosis in osteoarthritis cultured human chondrocytes. Osteoarthritis Cartilage. 2006 Jul;14(7):660-9.

47. Furman BD, Mangiapani DS, Zeitler E, Bailey KN, Horne PH, Huebner JL, et al. Targeting pro-inflammatory cytokines following joint injury: acute intra-articular inhibition of interleukin-1 following knee injury prevents post-traumatic arthritis. Arthritis Res Ther. 2014 Jun 25;16(3):R134.

48. Bigoni M, Sacerdote P, Turati M, Franchi S, Gandolla M, Gaddi D, Moretti S, Munegato D, Augusti CA, Bresciani E, Omeljaniuk RJ, Locatelli V, Torsello A: Acute and late changes in intraarticular cytokine levels following anterior cruciate ligament injury. J Orthop Res 2013, 31:315-321

49. Cameron M, Buchgraber A, Passler H, Vogt M, Thonar E, Fu F, Evans CH: The natural history of the anterior cruciate ligament-deficient knee. Changes in synovial fluid cytokine and keratan sulfate concentrations. Am J Sports Med 1997, 25:751-754. 
50. Marks PH, Donaldson ML: Inflammatory cytokine profiles associated with chondral damage in the anterior cruciate ligament-deficient knee. Arthroscopy 2005, 21:1342-1347.

51. Elsaid KA, Fleming BC, Oksendahl HL, Machan JT, Fadale PD, Hulstyn MJ, et al. Decreased lubricin concentrations and markers of joint inflammation in the synovial fluid of patients with anterior cruciate ligament injury. Arthritis Rheum 2008, 58:1707-1715.

52. Ward BD, Furman BD, Huebner JL, Kraus VB, Guilak F, Olson SA: Absence of posttraumatic arthritis following intraarticular fracture in the MRL/MpJ mouse. Arthritis Rheum 2008, 58:744-753.

53. Livshits G, Zhai G, Hart DJ, Kato BS, Wang H, Williams FM, et al.Interleukin-6 is a significant predictor of radiographic knee osteoarthritis: The Chingford Study. Arthritis Rheum. 2009 Jul;60(7):2037-45.

54. Stannus O, Jones G, Cicuttini F, Parameswaran V, Quinn S, Burgess J, et al. Circulating levels of IL-6 and TNF- $\alpha$ are associated with knee radiographic osteoarthritis and knee cartilage loss in older adults. Osteoarthritis Cartilage. 2010 Nov;18(11):1441-7.

55. Helmark IC, Mikkelsen UR, Børglum J, Rothe A, Petersen MC, Andersen O, et al. Exercise increases interleukin-10 levels both intraarticularly and peri-synovially in patients with knee osteoarthritis: a randomized controlled trial. Arthritis Res Ther. 2010;12(4):R126.

56. Kaneva MK, Kerrigan MJ, Grieco P, Curley GP, Locke IC, Getting SJ. Chondroprotective and anti-inflammatory role of melanocortin peptides in TNF- $\alpha$ activated human C-20/A4 chondrocytes. Br J Pharmacol. 2012 Sep;167(1):67-79.

57. Bálint G, Szebenyi B. Hereditary disorders mimicking and/or causing premature osteoarthritis. Baillieres Best Pract Res Clin Rheumatol 2000; 14:219-50;

58. Lagier R. Ochronotic arthropathy, an approach to osteoarthritis bone remodelling. Rheumatol Int. 2006; 26:561-4.

59. Braconi D, Laschi M, Taylor AM, Bernardini G, Spreafico A, Tinti L, et al. Proteomic and redox-proteomic evaluation of homogentisic acid and ascorbic acid effects on human articular chondrocytes. J Cell Biochem. 2010 Nov 1;111(4):922-32.

60. Mathy-Hartert M, Hogge L, Sanchez C, Deby-Dupont G, Crielaard JM, Henrotin Y. Interleukin-1beta and interleukin-6 disturb the antioxidant enzyme system in bovine 
chondrocytes: a possible explanation for oxidative stress generation. Osteoarthritis Cartilage. $2008 \mathrm{Jul} ; 16(7): 756-63$.

61. Albataineh E, Al-Sabou M, Al-Sarayreh S, Al-Tarawneh I, Alnawaiseh N. Levels of proInflammatory Mediators CRP, IL-1 $\beta$ and IL-6 in Alkaptonuria Patients. Journal of Biology and Life Science, 2014, Oct;6(1): 37-46.

62. Spreafico A, Milluci L, Ghezzi L, Geminiani M, Braconi D, Amato L, et al. Antioxidants inhibit SAA formation and pro-inflammatory cytokine release in a human cell model of alkaptonuria. Rheumatology (Oxford). 2013 Sep;52(9):1667-73.

63. J. B. Mistry, D. J. Jackson, M. Bukhari, A. M. Taylor. Osteoarticular cells tolerate shortterm exposure to nitisinone-implications in alkaptonuria. Clin Rheumatol. 2015 May 31. [Epub ahead of print]

\section{Figure Legends}

Figure 1: Mean number of pigment deposits in 10 day (left) and 21 day (right). C20/A4 cell cultures supplemented with HGA and IL-1 $\beta$. Pigment counts from x10 magnification images, expressed as the number of deposits per unit area. Results are the mean of 6 fields from 6 coverslips. Error bars signify the SEM. Statistically significant differences between groups denoted by $* * \mathrm{p}<0.01$ and $* * * \mathrm{p}<0.001$.

Figure 2: Mean number of viable cells in 10 day C20/A4 cell cultures supplemented with HGA and IL-1 $\beta$. Number of viable cells expressed as the number of cells per ml. Results are the mean of 5 counts from each of 6 wells. Error bars signify the SEM. Statistically significant differences between groups denoted by $* * * \mathrm{p}<0.001$.

Figure 3: Mean number of pigment deposits in 10 day (left),and 23 day (right). C20/A4 cell cultures supplemented with HGA and IL-6. Pigment counts from x10 magnification images, expressed as the number of deposits per unit area. Results are the mean of 6 fields from 6 coverslips. Error bars signify the SEM. Statistically significant differences between groups denoted by $* \mathrm{p}<0.05, * * \mathrm{p}<0.01$ and $* * * \mathrm{p}<0.001$.

Figure 4: Mean number of viable cells in 10 day C20/A4 cell cultures supplemented with HGA and IL-6. Number of viable cells expressed as the number of cells per ml. Results are the mean of 5 counts from each of 6 wells. Error bars signify the SEM. Statistically significant differences between groups denoted by $* * * p<0.001$. 
Figure 5: Mean number of pigment deposits in 10 day (left) and 21 day (right). C20/A4 cell cultures supplemented with HGA and IL-10. Pigment counts from x10 magnification images, expressed as the number of deposits per unit area. Results are the mean of 6 fields from 6 coverslips. Error bars signify the SEM. Statistically significant differences between groups denoted by $* \mathrm{p}<0.05, * * \mathrm{p}<0.01$ and $* * * \mathrm{p}<0.001$.

Figure 6: Mean number of viable cells in 10 day C20/A4 cell cultures supplemented with HGA and IL-10. Number of viable cells expressed as the number of cells per ml. Results are the mean of 5 counts from each of 6 wells. Error bars signify the SEM. Statistically significant differences between groups denoted by $* * * \mathrm{p}<0.001$. 